\title{
PARADIGMA SISTÊMICO NO DESENVOLVIMENTO HUMANO E FAMILIAR: A TEORIA BIOECOLÓGICA DE URIE BRONFENBRENNER
}

\author{
SYSTEMIC PARADIGM WITHIN HUMAN DEVELOPMENT AND FAMILY: URIE BRONFENBRENNER'S \\ BIOECOLOGICAL THEORY \\ PARADIGMA SISTÉMICO EN EL DESARROLLO HUMANO Y FAMILIAR: LA TEORÍA \\ BIOECOLÓGICA DE URIE BRONFENBRENNER
}

André de Carvalho Barreto*

\begin{abstract}
Resumo
Este ensaio objetiva apresentar e discutir conceitos da teoria bioecológica de Urie Bronfenbrenner, que é fundamentada no paradigma sistêmico e na perspectiva do curso de vida aplicada ao estudo do desenvolvimento familiar e humano. Sua teoria compreende o desenvolvimento como um fenômeno de continuidade e de mudança das características biopsicológicas dos seres humanos. Além de reconhecer a importância dos contextos mais próximos e remotos no desenvolvimento, ele enfatizou tanto a passagem do tempo, os níveis estrutural e funcional da pessoa quanto o processo das interaçóes organismo e ambiente. Seus estudos asseveram ainda que as relaçóes bidirecionais devem abranger, além das díades, outras pessoas na interação. Considera-se que, apesar da notória contribuição dos estudos do autor, os conceitos de sua teoria bioecológica e o modelo processo, pessoa, contexto e tempo necessitam avançar teórica e metodologicamente.
\end{abstract}

Palavras-chave: Teoria bioecológica. Psicologia do desenvolvimento. Paradigma sistêmico. Ciência do desenvolvimento humano. Ecologia do desenvolvimento humano.

\begin{abstract}
The following essay aims at the presentation and discussion of concepts from Urie Bronfenbrenner's Bioecological Theory, used as a systemic approach applied to the study of human and family development. The most important concepts of his Theory and its process-person- context-time model are discussed by pointing to their relevance in fixing a solid systemic theory that advances the given areas of knowledge, although they intend to deal with the area of human development and in particular with family
\end{abstract}

\footnotetext{
Texto recebido em 30 de julho de 2013 e aprovado para publicação em 14 de julho de 2014.

Doutorando em Processos de Desenvolvimento Humano e Saúde pela Universidade de Brasília e em Psicologia Social pela Universidade Ruhr de Bochum (Alemanha). Bolsista CAPES e DAAD. Endereço: Wiemelhauser Str., 180, Bochum, Alemanha. CEP: 44799. E-mail: andrecarvalhobarreto@yahoo.com.br.
} 
development. It is claimed that Bronfenbrenner's findings have been useful - and are still useful - for grounding several investigation and theoretical models, which already allowed understanding of psychological processes beyond Psychology of Development and which furthermore help to relate theory to its application in a better way.

Keywords: Bioecological theory. Family development. Systemic paradigm. Developmental science. Ecology of human development.

\section{Resumen}

Este trabajo tiene como objetivo presentar y discutir los conceptos de la teoría bioecológica de Urie Bronfenbrenner, basada en el paradigma sistémico y en la perspectiva del ciclo vital aplicado al estudio del desarrollo humano y de la familia. Su teoría incluye el desarrollo como un fenómeno de continuidad y de cambio de las características biopsicológicas humanas. Además de reconocer la importancia de los contextos próximos y remotos en el desarrollo, enfatizó tanto el paso del tiempo, los niveles estructurales y funcionales de la persona, como el proceso de las interacciones entre el organismo y el ambiente. Sus estudios afirman también que las relaciones bidireccionales deben abarcar, además de las diadas, otras personas en la interacción. Se considera que a pesar de la notoria contribución de los estudios el autor, los conceptos de su teoría bioecológica y el modelo proceso, persona, contexto y tiempo, requieren avanzar teórica y metodológicamente.

Palabras clave: Teoría bioecológica. Psicología del desarrollo. Paradigma sistémico. Ciencia del desarrollo humano. Ecología del desarrollo humano.

\section{INTRODUÇÃO}

O s estudos de Urie Bronfenbrenner (1917-2005), apresentados nos seus 14 livros e mais de 300 artigos e capítulos, serviram como fundamento para várias investigações por diversas instituiçõoes (e.g., Organização Mundial da Saúde) e disciplinas: Educação Física, Enfermagem, Fisioterapia, Gerontologia, Oncologia, Pediatria, Psicologia, Saúde Coletiva, Terapia Ocupacional, entre outras. Essa base tem sido utilizada também para compreensão de diversos fenômenos, entre eles a paretalidade, resiliência, uso de drogas, violência, dentre outras.

Um estudo de revisão da literatura nacional sobre parentalidade (CarvalhoBarreto, 2013), por exemplo, identificou serem os estudos de Bronfenbrenner um dos mais utilizados para compreender esse fenômeno. No entanto, a leitura dessas produções identificou discrepância na interpretação de sua teoria. Essas diferenças de interpretação e nomenclaturas da teoria de Bronfenbrenner 
podem ter sido ocasionadas, provavelmente, por uma interpretação parcial de sua produção pela leitura de apenas um de seus trabalhos. Este estudo teórico objetiva, portanto, trazer e discutir conceitos da teoria bioecológica de Urie Bronfenbrenner, na qualidade de uma teoria sistêmica aplicada ao estudo do desenvolvimento familiar e humano.

A ausência de uma perspectiva sistêmica para compreensão do desenvolvimento da pessoa é percebida no estudo realizado por Looft (1972). Nele, são analisados os principais tratados em Psicologia do Desenvolvimento entre as décadas de 1930 e 1970. Este estudo indica uma disciplina essencialmente positivista naquele período, centrada, especialmente, em investigaçōes sobre a criança e o adolescente, e na covariação entre estímulos controlados e respostas.

As investigações sobre interações no desenvolvimento familiar na época de 1930 e 1970 focavam a compreensão unidirecional das relações de causa e efeito de como a mãe afeta o desenvolvimento do bebê (Bronfenbrenner, 1977b; Newcome, 1999). Dada a ênfase no estudo do desenvolvimento infantil, eram raros os estudos que avançassem além da díade mãe-bebê e alcançassem todo o sistema familiar (Bronfenbrenner, 2011). Assim, permaneceram como exceções as investigaçóes que apontavam a bidirecionalidade, a interdependência e $o$ caráter sistêmico do desenvolvimento humano e familiar até a década de 1970 (Hinde, 1979).

O paradigma sistêmico teve origem após a Segunda Guerra Mundial, em 1950, sob influência de Ludwig von Bertalanffy (1901-1972). Biólogo austríaco, von Bertalanffy considerava limitado o poder explicativo do paradigma positivista sobre os organismos por deter as características deterministas e unidirecionais; diferentemente, o organismo é um sistema complexo, constituído por vários elementos interconectados, que devem ser compreendidos em suas particularidades e correlatamente (Vasconcelos, 2002), como totalidade.

Bronfenbrenner tem a originalidade de trazer esse novo modelo de compreensão dos organismos vivos à explicação dos processos de mudança e na linha do tempo, que são objetos da Psicologia do desenvolvimento (Schaffer \& Kipp, 2012). Alinhada ao paradigma positivista, a investigação do desenvolvimento infantil caracterizou-se pela realização de "experimentos" em condições controladas, convertendo-se na "ciência do comportamento desconhecido da criança em situações desconhecidas com adultos desconhecidos pelos períodos de tempo mais breves possíveis" (Bronfenbrenner, 1977b, p. 513). Os resultados desses achados, muitas vezes, não representavam a realidade do desenvolvimento, pois negavam ao ser humano e à família elementos interconectados que os afetavam direta e indiretamente. 
Para compreender o paradigma positivista de acordo com os modelos psicológicos, Lewin (1975) estabeleceu relação desses modelos com conceitos da física aristotélica ou teoria de classe. Lewin assevera que, conforme a teoria de classe, os fenômenos psicológicos são explicados, muitas vezes, pela regularidade que o comportamento se apresenta, classificando-os dentro de padrôes, sendo de propriedade fortuita casos particulares. $\mathrm{O}$ fato de crianças, por exemplo, serem costumeiramente negativas aos três anos constataria que o negativismo é inerente à natureza do infante. Assim, o desenvolvimento é explicado pelas categorias que lhes são atribuídas, sem buscar compreender os processos pelos quais ele possa ser causado (Bronfenbrenner, 1977a).

Fundamentado nos pressupostos da teoria de classe apresentados por Lewin (1975), Bronfenbrenner (1988) estabelece as investigaçôes positivistas do desenvolvimento humano em quatro modelos, a saber:
a) endereço social;
b) nicho sociológico;
c) atributos pessoais; e
d) pessoa-contexto.

O modelo do endereço social, originado pelos estudos de Schwabe e Bartholomai (1870), era o mais empregado para compreender como os contextos sociais (a exemplo da moradia, classe social, nacionalidade, grupo étnico) afetam o desenvolvimento. Entretanto, nesse modelo, cada contexto social era investigado em separado, tratado como variável relacionada ao desenvolvimento e não como condição de desenvolvimento, tal como passa a ocorrer na abordagem sistêmica dos fenômenos.

O modelo do nicho sociológico permitiu compreender a interação de dois ou mais endereços sociais sobre o desenvolvimento e possibilitou, ainda, identificar localizações sociais favoráveis ou desfavoráveis ao resultado do desenvolvimento. $\mathrm{O}$ dos atributos pessoais buscava compreender como a interferência das características biológicas e físicas, como idade, sexo e estado fisiológico do organismo, interferem nos resultados do desenvolvimento (Bronfenbrenner, 1988).

O último, pessoa-contexto, perpassava as categorias dos modelos do endereço social e dos atributos pessoais, constituindo uma compreensão mais ampla sobre 
o desenvolvimento. Entretanto, apesar de esse último modelo integrar diversos elementos na análise, ele não deve ser confundido com um modelo sistêmico, pois lhe falta a compreensão do efeito dos processos nos quais as propriedades da pessoa e do ambiente (isolados ou combinados) funcionam na produção de resultados no desenvolvimento humano (Bronfenbrenner, 1988; Bronfenbrenner \& Evans, 2000).

As características do paradigma sistêmico pensados por von Bertlanffy de compreender os processos dos fenômenos e relacioná-los dinamicamente com outros eram capazes de ir além dos modelos aristotélicos. Tais características possibilitaram não apenas explicar fenômenos ou encontrar soluçōes, mas também ajudar a produzir teorias que rompessem com o paradigma positivista e os modelos da teoria de classe, criando condições empíricas sólidas de produzir conhecimentos multi e interdisciplinares (Vasconcelos, 2002).

O empreendimento de Bronfenbrenner não foi isolado. Ao longo das décadas de 1970 e 1980, a inserção do paradigma sistêmico no estudo do desenvolvimento humano e familiar ocorreu de formas diversas (Dessen, 1997; Schaffer \& Kipp, 2012). Pesquisadores biocomportamentais passaram a incluir o paradigma de von Bertalanffy no estudo da embriologia do comportamento e da biologia comportamental (Cairns, Elder \& Costello, 1996). Da mesma forma, estudiosos partiram dos modelos holísticos de Kurt Lewin e os integraram na compreensão do efeito do meio no desenvolvimento humano (Tudge, Gray \& Hogan, 1997). Destacam-se ainda os teóricos sistêmicos da família, que investigaram a relação entre o ciclo vital dos membros da família e o desenvolvimento familiar (Carter \& McGoldrick, 1989/2001). Mas, de acordo com Lerner (2005/2011), Bronfenbrenner não somente presenciou o aumento da ênfase sistêmica na pesquisa do desenvolvimento, mas também foi seu principal articulador e suas ideias as mais citadas em todas as teorias sistêmicas do desenvolvimento humano, que contribuíram para romper em definitivo com o paradigma positivista.

Lerner (2005) e Ceci (2006) argumentam, ainda, que Bronfenbrenner foi igualmente o principal líder na consolidação da Ciência do Desenvolvimento, ao ressaltar a relação entre teoria e aplicação; pesquisa e prática; e contribuir para uma ciência que saía dos laboratórios para operar a intervenção e que passava a fundamentar outras áreas psicológicas, como a Psicologia Cultural (Rogoff, 2005).

A evolução de sua teoria e modelo sistêmico ocorre consoante ao seu desenvolvimento humano e científico. Isso é percebido no estudo sistemático de suas obras, razão por que a leitura de somente um artigo, capítulo ou volume 
de sua autoria enseje compreensão fragmentada, incompleta e, algumas vezes, equivocada de sua teoria, a qual se forma progressivamente. Didaticamente, essa evolução pode ser dividida em três fases.

A primeira, a da juventude, dura de 1942 até 1970. Neste período, Bronfenbrenner envolve-se com práticas de políticas públicas, aprofundando seus estudos sobre personalidade (Bronfenbrenner, 1960) e pesquisas transculturais, especialmente com estudos comparativos entre as sociedades estadunidense e soviética. Esse período pode ser considerado como o de sua transição científica de investigaçôes de teoria de classe para sistêmica. O cume dessa fase está no volume Two worlds of childhood: US and USSR (Bronfenbrenner, 1970).

$\mathrm{Na}$ sua segunda fase (vida adulta), de 1971 até 1980, Bronfenbrenner (1996) consolida seu modelo ecológico pelo livro A Ecologia do Desenvolvimento Humano: experimentos naturais e planejados, considerado por todos os cientistas do desenvolvimento como um marco mundial no estudo sistêmico sobre desenvolvimento humano. Nele se encontram os principais preceitos de seus modelo e teoria ecológicos.

Por fim, na fase da velhice, de 1981 até 2005, Bronfenbrenner clarifica sua insatisfação com as contribuições de sua proposta de 1996 para teoria, pesquisa e aplicações em política pública, amadurecendo suas ideias, consolidando-as como teoria bioecológica. No mesmo período, ele indica também sua preocupação com as futuras gerações ante contextos cada vez mais caóticos na sociedade mundial. Suas duas principais obras nessa fase foram: The State of Americans: this generation and the next (Bronfenbrenner, McClelland, Wethington, Moen \& Ceci, 1996) e Bioecologia do Desenvolvimento Humano: tornando os seres humanos mais humanos (2011), sendo a última uma atualização e compilação de suas principais produções que refletem sumariamente as bases e a evolução de sua teoria sistêmica.

Em suma, os estudos de Urie Bronfenbrenner, especialmente nas suas segunda e terceira fases, são uma evolução da teoria sistêmica para o estudo do desenvolvimento familiar e humano (Schaffer \& Kipp, 2012), pois o desenvolvimento deixou de ser definido como estudo das mudanças relacionadas à idade (Biaggio, 2011) e passou a configurar um "fenômeno de continuidade e de mudança das características biopsicológicas dos seres humanos como indivíduos e grupos" (Bronfenbrenner, 2001b, p. 6966). Nessa perspectiva, o desenvolvimento humano e familiar são faces de uma mesma moeda, estendendose ao longo do curso de vida e do desenvolvimento humano, englobando as várias gerações e a história da humanidade. 


\section{CURSO DE VIDA E DESENVOLVIMENTO HUMANO: INTERAÇÕES E DINÂMICA}

Inicialmente, é necessário clarificar os conceitos de:

a) ciclo de vida;

b) "lifespan";

c) estágios de vida;

d) fase de vida;

e) história de vida; e

f) curso de vida, que, muitas vezes, são utilizados de forma indiscriminada e equivocada por estudiosos (Elder, 1996; Newcome, 1999; Schaffer \& Kipp, 2012). Cada um desses merece atenção por sua relação com aspectos de continuidade e mudança, importantes no mapeamento conceitual da teoria de Bronfenbrenner.

O ciclo de vida, ou ciclo vital, frequentemente, descreve uma sequência de eventos ontogenéticos transcorridos do nascimento até a morte da pessoa e, ou, da família (Elder, 1996), destacando-se, como exemplo, o já mencionado ciclo vital utilizados pelos teóricos sistêmicos da família (Carter \& McGoldrick, 1989). Esse conceito foi por anos confundido com "lifespan", provavelmente pela inadequação teórica de tradução do livro Lifespan development, de Helen Bee (1994), que teve, por exemplo, seu título alterado para $O$ ciclo vital (Bee, 1997).

Ao longo da vida, ou, no inglês, "lifespan", refere-se à compreensão do desenvolvimento humano de forma contínua, multidimensional e multidirecional (Boyd \& Bee, 2011). O estudo do desenvolvimento ao longo da vida pode ocorrer com todos os estágios de desenvolvimento ou apenas alguns deles. A compreensão do "lifespan" é encontrada especialmente na teoria de Paul Baltes sobre o envelhecimento humano (Baltes, Staudinger \& Lindenberger, 1999).

O outro conceito bastante empregado é o de estágios de vida, ou estágios de desenvolvimento, que compreende o desenvolvimento como sendo constituído progressivamente, de ordem invariável, por estágios ou etapas sequenciais e hierárquicos, mas não necessariamente em intervalos de tempo fixos (Newcome, 1999), como no desenvolvimento psicossocial de Erik Erikson (1972). Ele surge 
como uma oposição ao conceito fase de vida que é empregado especialmente na descrição do desenvolvimento psicossexual, segundo a abordagem psicodinâmica; entende que as pessoas passam, ao longo da vida, por momentos com características normativas e que a vivência dessas fases contribui para a formação de uma estrutura psíquica definida em torno da mobilidade entre fases ou a fixação em uma ou mais delas (Newcome, 1999; Schaffer \& Kipp, 2012).

A concepção de história de vida remete ao desenvolvimento humano conforme percebido pelo sujeito em desenvolvimento, que reconstrói a cronologia dos eventos e atividades ocorrentes na sua vida, considerando o significado das experiências ocorridas nesse período (Elder, 1996; Reese, Yan, Jack \& Hayne, 2010). Por essa característica, o termo história de vida é também utilizado como um método de pesquisa do desenvolvimento humano, ele se baseia em autorrelatos livres ou dirigidos pelo pesquisador (Bertaux, 1981).

Por fim, o curso de vida, para Elder (1996), incorpora noções de tempo, contexto e processo no desenvolvimento, considerando as mudanças e interdependências da pessoa vinculadas à sua idade (Dessen \& Guedea, 2005). Esse é um dos conceitos essenciais nos estudos de Bronfenbrenner (2011) sobre trajetória de vida. A noção de trajetória pode ser ilustrada como diversos vetores que representam direçôes coexistentes de orientaçôes psicológicas. Ela se refere às tendências, mas não certezas, da pessoa em desenvolvimento em persistir em determinados padrões de conduta que a direcionam para um destino de vida (Sato, Hidaka \& Fukuda, 2009).

A experiência dentro do curso de vida é um elemento crítico na teoria de Bronfenbrenner. $O$ termo é usado não pela predileção por fundamentação filosófica existencialista (Bronfenbrenner, 2001b), mas pela ciência de que a esfera subjetiva dos sentimentos surge no curso de vida desde os primeiros meses de existência e se transforma ao longo dele, sem deixar de interferir nas trajetórias de vida. Assim, não apenas as propriedades objetivas são relevantes, mas também as experienciadas subjetivamente pelas pessoas em suas interações bidirecionais com outras pessoas e com o ambiente (Bronfenbrenner, 2011).

As interaçôes bidirecionais podem ser delimitadas como de primeira, segunda e terceira ordem, de acordo com Bronfenbrenner, McClelland, Wethington, Moen \& Ceci (1996); Bronfenbrenner (2001b). As de primeira ordem são constituídas pelas relações recíprocas e regulares entre uma díade, que promovem mudanças no curso de vida de ambos os envolvidos. As díades são as interações mais frequentemente investigadas na Ciência do Desenvolvimento. No entanto, para ser sistêmica, uma teoria deve considerar também os efeitos sobre o desenvolvimento dos sistemas $\mathrm{N}+2$, ou seja, as tríades, tétrades e relações mais amplas (Bronfenbrenner et al., 1996). 
Os efeitos de segunda e terceira ordem envolvem, assim, pelo menos uma terceira pessoa na interação com a díade. A diferença entre os efeitos de segunda ordem e os de terceira ordem está no contexto em que a terceira pessoa se encontra. No efeito de segunda ordem, as relações recíprocas e regulares entre a díade e a terceira pessoa ocorrem dentro do mesmo ambiente. O efeito de terceira ordem consiste em relações da tríade ou tétrade em dois ou mais ambientes diferentes. Por exemplo, uma díade formada por mãe e bebê está em casa. A terceira pessoa, como o filho mais velho, está na escola, e uma quarta pessoa, como o pai, encontra-se no trabalho. Esse mesmo princípio é aplicado para compreender as relaçôes entre contextos em que um sistema externo ao microssistema familiar, como escola ou local de trabalho dos pais, pode afetar o curso de vida da pessoa, mesmo que ela não esteja nesse sistema ambiental (Bronfenbrenner, 1985).

\section{SISTEMA AMBIENTAL: ECOLOGIA DO DESENVOLVIMENTO HUMANO}

A perspectiva ecológica é utilizada em diversas disciplinas, em especial, pela Biologia, para compreender os efeitos causados por eventos externos para a organização e transformação do organismo. O zoologista e evolucionista alemão Ernest Haeckel, em 1873, é o primeiro a propor essa nova ciência para estudar os organismos em seu ambiente (Tudge et al., 1997).

A ideia de estudar o desenvolvimento humano considerando o contexto surge paralelamente à ecologia de Haeckel, na década de 1870, também na Alemanha. Tal ideia é proposta por Schwabe e Bartholomai, que investigam a influência da vizinhança no desenvolvimento de crianças. São, no entanto, James Baldwin, John Dewey, George Herbert Mead e James Gibson, nos Estados Unidos, e Lev Vygotsky, na Bielorrússia, os primeiros teóricos a enfatizar a relevância do contexto na própria constituição dos fenômenos psicológicos (Ratner, 2002; Tudge et al., 1996).

O modelo ecológico ganha corpo na Psicologia do desenvolvimento quando Bronfenbrenner (1989) propõe a Ecologia do Desenvolvimento Humano, definida como

O estudo científico da progressiva acomodação mútua, durante todo o curso de vida, entre um ser humano ativo em crescimento e as propriedades em mudança nos contextos imediatos os quais a pessoa em desenvolvimento vive; nesse processo ela é afetada pelas relaçóes entre esse contexto imediato e os distantes, estando todos estes contextos encaixados (p. 187). 
A Ecologia do Desenvolvimento Humano difere de outras disciplinas que também têm o contexto como foco de análise, como a Psicologia Social, Ciências Sociais e Antropologia, por considerar relevantes aspectos biológicos e genéticos na compreensão do fenômeno do desenvolvimento humano e familiar, sendo este uma convergência entre as ciências biológica, psicológica e sociais (Bronfenbrenner et al., 1996). Todavia, devido à amplitude de sua proposta sistêmica de compreensão da relação pessoa-contexto, pesquisadores de áreas distintas à Psicologia do Desenvolvimento têm usado sua perspectiva (Zillmer, Schwartz, Muniz \& Meincke, 2011).

Ilustrativamente, Bronfenbrenner et al. (1996) representa seu modelo ecológico como um conjunto de quatro bonecas russas encaixadas uma na outra, no qual cada uma equivale a um sistema que, apesar de específico em suas características, é dinâmico e entrelaçado. Embora didática, a imagem das bonecas russas é hoje objeto de crítica entre interlocutores de Bronfenbrenner por dar pouco destaque às interações bidirecionais entre os subsistemas (Tudge, 2008).

O sistema mais interno (o microssistema) é o ambiente imediato que contém a pessoa, sendo esse sistema o "centro de gravidade" de toda sua teoria (Bronfenbrenner \& Moris, 1998, p. 1013). No microssistema, a pessoa experiencia posiçôes, relacionamentos e atividades em relaçôes face a face com outras pessoas em desenvolvimento, como com os aspectos físicos e simbólicas desse ambiente. Para que esse sistema promova mudanças no seu curso de vida, as pessoas presentes devem ter temperamentos, personalidades e sistemas de crenças distintas (Bronfenbrenner, 1989).

O sistema ulterior, o mesossistema, representa as ligações e processos entre dois ou mais microssistemas nos quais a pessoa efetiva seu curso de vida, tais como as relações entre casa-escola e casa-trabalho. A diferença desse nível ecológico para seu subsequente, o exossistema, é que, neste último, a pessoa normalmente não está presente nesse ambiente, mas os eventos que nele ocorrem interferem indiretamente no microssistema no qual a pessoa está (Bronfenbrenner et al., 1996; 1989).

Importante ressaltar que a classificação de espaços ou instituições, grupos ou pessoas de acordo com esses sistemas não é rígida. Por serem dinâmicos, um pode se converter em outro, a depender da variedade de fatores internos ou externos à pessoa em desenvolvimento. Essa diferença é destacada no estudo de Carvalho-Barreto, Vidal e Bucher-Maluschke (2004), no qual uma criança vítima de violência no seu microssistema familiar teve no abrigo seu exossistema, pois, apesar de ela nunca ter estado nele antes da denúncia de violência, ele interferiu indiretamente no seu desenvolvimento, pois tinha profissionais que 
forneciam apoio social à criança. No momento, contudo, em que ela passa a viver no abrigo, ocorre uma mudança de sistema na qual o abrigo passa a ser seu microssistema, e a família, o mesossistema. Além dessas mudanças de sistemas, os próprios eventos normativos de desenvolvimento (e.g., saída da casa dos pais depois do casamento) favorecem reconfiguraçôes de sistemas, sendo nomeados por Bronfenbrenner et al. (1996; 1994) como transição ecológica.

O macrossistema, nível mais amplo do sistema ambiental, é compreendido como o contexto que engloba qualquer sociedade ou grupo social, sua cultura, subcultura ou outra estrutura social maior. Pessoas nesse mesmo sistema compartilham valores, crenças, estilos de vida, recursos, trajetórias de vida, oportunidades e padrões de intercâmbio social. Junto com o microssistema, Bronfenbrenner (1989) atribui relevância especial aos macrossistemas como geradores de resultados no desenvolvimento, por conterem os outros níveis micro, meso e exossistêmicos, influenciando e sendo influenciado por todos eles (Tudge, 2008).

Idealizado entre as décadas de 1970 e 1980, a Ecologia do Desenvolvimento Humano, ao longo dos anos que se seguiram, consolidou-se na Ciência do Desenvolvimento, sendo referendada nos principais tratados sobre Desenvolvimento Humano (Schaffer \& Kipp, 2012). Contudo, Bronfenbrenner (2001b) começou a perceber a lacunas em suas ideias, propondo uma nova teoria do desenvolvimento humano próximo ao fim de sua vida: a teoria bioecológica.

\section{EMERGÊNCIA DA TEORIA BIOECOLÓGICA}

Nos trabalhos mais recentes (Bronfenbrenner \& Evans, 2000; Bronfenbrenner 2001b; 2005; Bronfenbrenner \& Morris, 1998, 2006), Bronfenbrenner atesta que sua perspectiva sistêmica no desenvolvimento humano e familiar está, ela própria, em desenvolvimento e introduz ampliaçôes. Passa a denominar sua teoria como bioecológica, concede mais ênfase à linguagem e aos símbolos na compreensão da interação pessoa-contexto e ressalta a importância dos processos proximais e do tempo.

A evolução do termo ecologia para bioecologia provém do reconhecimento de Bronfenbrenner da incompletude de suas ideias anteriores, passando a enfatizar os níveis estrutural e funcional da pessoa (i.e., aspectos biológicos, cognitivos, emocionais e comportamentais), incluindo-os no cerne dos sistemas ecológicos criados previamente (Bronfenbrenner \& Morris, 1998, 2006).

Outro alargamento da perspectiva de Bronfenbrenner foi a inclusão dos símbolos e da linguagem, isto é, do sistema semiótico, presente nas interações 
da pessoa em seu microssistema (Lerner, 2005). Esse alargamento inclui a compreensão de que as intenções, objetivos e açōes ocorrentes no desenvolvimento familiar e humano são originadas além das interações entre pessoas, mas também com os fenômenos culturais presentes em seu contexto imediato.

Somado a isso, em suas investigaçōes, Bronfenbrenner (1988; Bronfenbrenner $\&$ Evans, 2000) indica que, além da pessoa e do contexto, o processo proximal pelo qual o desenvolvimento é realizado é condição "sine qua non" para o desenvolvimento humano e familiar. O processo proximal representa as formas de interação do organismo com o ambiente, que ocorrem regularmente, sendo ele a forma, a força, o conteúdo e a direção dos resultados no desenvolvimento humano (Bronfenbrenner \& Evans, 2000). Exemplos de como o processo proximal interfere nas trajetórias de vida podem ser encontrados nas relações bidirecionais do conforto do bebê com sua mãe e nas relações semióticas de aprendizagem de novas habilidades (Bronfenbrenner, 2001b).

A compreensão da particular relevância dos processos proximais no desenvolvimento acarretou a ampliação da influência da pessoa nos resultados do desenvolvimento. Bronfenbrenner e Morris (1998) identificam três características da pessoa como intervenientes na direção das trajetórias de vida, pelo fato de promoverem a direção e força dos processos proximais. A primeira se refere às disposiçôes comportamentais ativas. Elas são as características pessoais generativas, ou seja, as habilidades das pessoas de se engajarem em atividades individuais ou com outras pessoas, comum em indivíduos comunicativos, ou as características pessoais inibidoras, que são o oposto das disposições ativas, presentes, por exemplo, naqueles com timidez excessiva.

A segunda, as características de recursos, representa as capacidades ou as necessidades da pessoa para o funcionamento efetivo dos processos proximais em determinado curso de seu desenvolvimento. Isto é, são aspectos orgânicos (como problemas genéticos, bebês desnutridos, malformação) que interferem nos processos proximais. Finalmente, as características de demanda são atributos pessoais que podem auxiliar ou dificultar reaçôes do contexto social, inibindo ou favorecendo os processos. Por exemplo, bebês felizes atraem mais afeto do que os agitados (Bronfenbrenner, 2011; Bronfenbrenner \& Morris, 1998, 2006; Elder, 1996).

Malgrado Bronfenbrenner (1988) compreender a relevância do tempo, ou cronossistema, na compreensão do impacto de eventos e experiências anteriores (isolada ou sequencialmente) no resultado do desenvolvimento, este se torna o último elemento acrescido tardiamente ao seu modelo. $\mathrm{O}$ cronossistema está dividido em três subsistemas: microtempo, mesotempo e macrotempo. Aos episódios de continuidade e descontinuidade do processo proximal, 
Bronfenbrenner e Morris (1998) nomearam microtempo. Ele se caracteriza como o espaço temporal do aqui e agora que ocorre com a pessoa que está em determinada atividade ou interação com outras pessoas, objetos ou símbolos no seu contexto mais imediato (Tudge, 2008).

O mesotempo se remete aos episódios em espaços temporais mais amplos, como dias, semanas, meses ou anos. Isto é, em que frequência as atividades e as interações da pessoa com outras pessoas, objetos e símbolos no ambiente imediato desta pessoa (Bronfenbrenner \& Morris, 1998, Tudge, 2008).

Por fim, o macrotempo refere-se às mudanças ocorridas na sociedade e na família pelas heranças inter e intrageracionais, afetando os processos proximais da pessoa durante todo seu curso de vida. Essas modificações ocorrem segundo os eventos históricos singulares (e.g., promulgação da Constituição brasileira, em 1988), quando as pessoas em desenvolvimento têm determinada idade (Bronfenbrenner \& Morris, 1998, Tudge, 2008). Estudos de coortes, como realizados por Elder $(1976,1996)$ sobre os efeitos da Grande Depressão nos Estados Unidos, que indicou modificações no curso de vida das pessoas que passaram por esse evento histórico marcante, deixam explícito o efeito do macrotempo ao longo do desenvolvimento humano e familiar.

Surge, portanto, o modelo processo, pessoa, contexto e tempo (PPCT) como elemento fundante da teoria bioecológica (Bronfenbrenner \& Morris, 1998, 2006). Em razão de a dinâmica desse modelo ser semelhante ao criado no sistema ecológico, ilustrações inapropriadas com anéis concêntricos encaixados como bonecas russas foram demasiadamente apresentadas na literatura para explicar o PPCT (Spesato, Valentini, Krebs \& Berleza, 2009).

Para Tudge (2008), todavia, o modelo PPCT se estende a esse tipo de expressão. $\mathrm{O}$ autor, apresenta com maior clareza o dinamismo sistêmico da teoria bioecológica, especialmente no que tange ao microssistema. Ele sai da estrutura rígida e pouco dinâmica das bonecas russas, apresentado em 1979. Apesar de didática, a estrutura das bonecas russas foge da perspectiva sistêmica e do próprio desenvolvimento da teoria de Bronfenbrenner.

\section{CONSIDERAÇÕES FINAIS: O FUTURO}

Por mais de 60 anos, o Urie Bronfenbrenner ou somente Urie, como era chamado pelos amigos e alunos, tornou-se referência de excelência profissional na área da Ciência do Desenvolvimento Humano que, em virtude de sua colaboração como de outros profissionais (David Magnusson, Erik Erikson, Glen Elder Jr., Gilbert Gettlieb, Helen Bee, Jean Piaget, Lev Vygotsky e Paul 
Baltes), tornou-se uma área multi e interdisciplinares (Cairms, Elder \& Costello, 1996; Lerner, 2005; Schaffer \& Kipp, 2012), podendo suas ideias ser aplicadas em disciplinas díspares da Psicologia, que, pela perspectiva sistêmica, possam explicar a complexa relação entre os aspectos biológicos, psicológicos e sociais.

A obra de Bronfenbrenner é marcada por uma preocupação ética, pela qual a produção científica deve resultar na constituição de conhecimentos que possam agregar valor tanto à Ciência do Desenvolvimento como à sociedade, acreditando ser possível "tornar mais humano o ser humano" (Bronfenbrenner, 1995, p. 117). Para o autor, os políticos e cientistas deveriam ter atenção especial às futuras gerações, em razão da organização caótica em que se encontram as sociedades, com aumento da delinquência juvenil, pobreza, entre outros eventos causadores da disfunção do desenvolvimento (Bronfenbrenner et al., 1996).

Bronfenbrenner, ao final de sua vida, desenvolve um modelo complexo (pessoa processo contexto tempo) capaz de promover mudanças sistêmicas positivas na ciência e na sociedade (Lerner, 2005). Entretanto a ausência de estudos empíricos realizados pelo próprio dificulta pensar como esse modelo poderia ser totalmente efetivado no propósito a que se destina. Tudge (2008) assinala, ainda, que a complexidade da integração e diferenciação dos elementos amplos e intrínsecos do PPCT dificulta sua efetividade como delineamento de pesquisa. Considera ele que, por ser muito abrangente, custoso e mais ajustado ao modelo cientificista estadunidense do que a outros contextos, surgem divergências notórias de sua aplicação entre pesquisadores de áreas diversas e países.

Além disso, talvez por não ter sido seu foco de investigação ou não ter tido tempo em vida, aspectos de sua teoria acabaram não sendo aprofundados, como a relação pessoa e contexto, como salienta Rogoff (2005). Outro exemplo está na relevância do sistema semiótico no desenvolvimento humano que, em muitas obras na fase da velhice, é ratificado, mas não aprofundado. Isso gera divergências notórias sobre a confirmação no uso das propostas de Bronfenbrenner entre os pesquisadores da Ciência do Desenvolvimento, como também de outras disciplinas, ocasionando posições divergentes se suas ideias constituiriam um modelo ou uma teoria científica.

A despeito das críticas à sua teoria, algumas feitas pelo próprio (Bronfenbrenner, 2001b), deve-se reconhecer que as contribuições e reflexões de Bronfenbrenner têm servido como fundamentação para diversas finalidades teóricas e de investigação (quantitativa, qualitativa e multimetodológica), possibilitando a compreensão do desenvolvimento de forma sistêmica e multidisciplinar, ao incluir novos pressupostos e guias de aplicação, que vão ao encontro do avanço científico e social por ele almejado, mas que, como toda teoria, também esta necessita avançar. 


\section{REFERÊNCIAS}

Baltes, P. B., Staudinger, U. M. \& Lindenberger, U. (1999). Lifespan psychology: theory and application to intellectual functioning. Annual Review of Psychology, 50, 471-507.

Bee, H. (1994). Lifespan development. New York: Harper Collins College Publishers.

Bee, H. (1997). O ciclo vital. R. Garcez (Trad.). Porto Alegre: Artes Médicas, (Publicado originalmente em 1994)

Bertaux, D. (1981). Biography and society: the life history approach in the social sciences. Beverly Hills, Calif: Sage Publications.

Biaggio, A. (2011). Psicologia do desenvolvimento. Petrópolis: Vozes, 2011. (Publicado originalmente em 1975)

Boyd, D. \& Bee, H. (2011). Lifespan development. New Jersey: Prentice Hall PTR.

Bronfenbrenner, U. (1960). Freudian theories of identification and their derivatives. Child Development, 31, 15-40.

Bronfenbrenner, U. (1970). Two words of childhood: USA and USSR. New York: Russell Sage Foundation.

Bronfenbrenner, U. (1977a). Lewinian space and ecological substance. Journal of Social Issues, 33(4), 199-212.

Bronfenbrenner, U. (1977b). Toward an experimental ecology of human development. American Psychologist, 32, 513-531.

Bronfenbrenner, U. (1985). The future of childhood. In V. Greaney (Ed.), Children: Needs and rights. (pp. 167-186). New York: Irvington Publishers.

Bronfenbrenner, U. (1988). Interacting systems in human development. Research paradigms: present and future. In N. Bolger, A. Caspi, G. Downey \& M. Moorehouse (Ed.), Persons in context: Developmental processes (pp. 2549). New York: Cambridge University Press.

Bronfenbrenner, U. (1989). Ecological systems theory. In R. Vasta (Ed.), Six theories of child development: revised formulations and current issues. (pp. 185246). Greenwich: JAI. 
Bronfenbrenner, U. (1994). Ecological models of human development. In T. Husten \& T. N. Postelethwaite, International encyclopedia of education (Vol. 3, pp. 1643-1647). New York: Elsevier Science.

Bronfenbrenner, U. (1995). Uma família e um mundo para o bebê XXI: sonho e realidade. In J. Gomes-Pedro \& M. F. Patrício, Bebé XXI: criança e família na viragem do século. (pp. 115-126). Lisboa: Fundação Calouste Gulbenkian.

Bronfenbrenner, U. (1996). A ecologia do desenvolvimento humano: experimentos naturais e planejados. M. A. V. Veronese (Trad.). Porto Alegre: Artmed. (Publicado originalmente em 1979)

Bronfenbrenner, U. (2001a). Growing chaos in the lives of children, youth, and families: how can we turn it around? In J. C. Westman (Ed.), Parenthood in America. (pp. 197-210). Madison: University of Wisconsin Press.

Bronfenbrenner, U. (2001b). The bioecological theory of human development. In N. J. Smelser \& P. B. Baltes (Eds.), International encyclopedia of the social and behavioral sciences (Vol. 10, pp. 6963-6970). New York: Elsevier.

Bronfenbrenner, U. (2011). Bioecologia do desenvolvimento humano: tornando os seres humanos mais humano. A. Carvalho-Barreto (Trad.). Porto Alegre: Artmed. (Publicado originalmente em 2005)

Bronfenbrenner, U. \& Evans, G. W. (2000). Developmental science in the 21st century: emerging questions, theoretical models, research designs and empirical findings. Social development, 9(1), 115-125.

Bronfenbrenner, U. \& Morris, P. A. (1998). The ecology of developmental process. In W. Damon \& R. M. Lerner (Eds.), Handbook of child psychology: theoretical models of human developmental. (Vol. 1, pp. 939-991). New York: John Wiley.

Bronfenbrenner, U. \& Morris, P. A. (2006). The bioecological model of human development. In W. Damon \& R. M. Lerner (Eds.), Handbook of child psychology: theoretical models of human developmental. (Vol. 1, pp. 793-828). New York: John Wiley.

Bronfenbrenner, U., McClelland, P., Wethington, E., Moen, P. \& Ceci, S. J. (1996). The state of Americans: this generation and the next. New York: Free Press. Cairns, R. B., Elder, G. H. \& Costello, E. J. (Orgs.), (1996). Developmental science. New York: Cambridge University Press. 
Carter, B. \& McGoldrick, M. (Orgs.). (1989). As mudanças no ciclo vital familiar: uma estrutura para a terapia familiar. M. A. V. Veronese (Trad.). Porto Alegre: Artmed, 2001. (Publicado originalmente em 1989)

Carvalho-Barreto, A. (2013). A parentalidade no ciclo de vida. Psicologia em Estudo, 18(1), 137-147.

Carvalho-Barreto, A., Vidal, A. A. \& Bucher-Maluschke, J. S. N. F. (2004). Agressor sexual na dinâmica relacional da família: um estudo de caso. In J. S. N. F. Bucher-Maluschke, G. Maluschke \& K. Hermanns (Coords), Direitos humanos e violência: Desafios da ciência e da prática. (pp. 197-212). Fortaleza: Fundação Konrad Adenauer.

Ceci, S. J. (2006). Urie Bronfenbrenner (1917-2005). American Psychologist, 61, 173-174.

Dessen, M. A. (1997). Desenvolvimento familiar: um sistema triádico para poliádio. Temas em Psicologia, 5(3), 51-61.

Dessen, M. A. \& Guedea, M. T. D. (2005). A ciência do desenvolvimento humano: ajustando foco de análise. Paidéia, 15(30), 11-20.

Elder, G. H. (1976). Children of the great depression. Chicago: University of Chicago.

Elder, G. H. (1996). Human lives in changing societies: life curse and developmental insights. In R. B. Cairns, G. H. Elder \& E. J. Costello (Ed.), Developmental science. (pp. 31-62). New York: Cambridge University Press.

Erikson, E. H. (1972). Identidade: juventude e crise. Rio de Janeiro: Zahar. (Publicado originalmente em 1968)

Hinde, R. A. (1979). Towards understanding relationships. Londres: Academic Press.

Lerner, R. M. (2011). Prefácio. In U. Bronfenbrenner, Bioecologia do desenvolvimento humano: tornando os seres humanos mais humanos. A. CarvalhoBarreto (Trad.). (pp. IX-XXV). Porto Alegre: Artmed. (Publicado originalmente em 2005)

Lewin, K. (1975). Teoria dinâmica da personalidade. A. Cabral (Trad.). São Paulo: Cultrix. (Publicado originalmente em 1935) 
Looft, W. R. (1972). The evolution of developmental psychology. Human Development, 15, 187-201.

Newcome, N. (1999). Desenvolvimento infantil: abordagem de Mussen. B. Buchweitz (Trad.) Porto Alegre: Artmed.

Ratner, C. (2002). Cultural psychology: Theory and method. New York: Kluwer.

Reese, E., Yan, C., Jack, F. \& Hayne, H. (2010). Emerging identities: narrative and self from early adolescence. In K. C. McLean \& M. Pasupathi, Narrative development in adolescence. (pp. 23-42). New York: Spring.

Rogoff, B. (2005). A natureza cultural do desenvolvimento humano. R. C. Costa (Trad.). Porto Alegre: Artmed.

Sato, T., Hidaka, T. \& Fukuda, M. (2009). Depicting the dynamic of living the life: The trajectory equifinality model. In J. Valsiner, P. C. M. Molenaar, M. C. D. P. Lyra \& N. Chaudhary, Dynamic process methodology in social and developmental sciences. (pp. 217-240). New York: Springer.

Schwabe, B., \& Bartholomai, F. (1870). Der Vorstellungskreis der Berliner Kinder beim Eintritt in die Schule. In Berlin und seine Emwickelung: Städtisches Jahrbuch für Volkswirthschaft und Statistik: Vierter Jahrgang. Berlin: Guttentag.

Schaffer, A. R. \& Kipp, K. (2012). Psicologia do desenvolvimento: infância e adolescência. São Paulo: Cengage.

Spesato, B. C., Valentini, N. C., Krebs, R. J. \& Berleza, (2009). Educação infantil e intervenção motora: um olhar a partir da teoria bioecológica de Bronfenbrenner. Movimento, 15(4), 147-173.

Tudge, J. (2008). A teoria de Urie Bromfenbrenner: uma teoria contextualista? In L. Moreira \& A. M. A. Carvalho (Org.), Família e educação: olhares da Psicologia. (pp. 209-231). São Paulo: Paulinas.

Tudge, J., Gray, J. T. \& Hogan, D. M. (1997). Ecological perspectives in human development: a comparison of Gibson and Bronfenbrenner. In J. Tudge, M. J. Shanahan \& J. Valsiner (Ed.), Comparisons in human development: understanding time and context. (pp. 72-105). New York: Cambridge University Press.

Vasconcelos, M. J. E. (2002). Pensamentos sistêmicos: o novo paradigma da ciência. Campinas: Papirus. 
Zillmer, J. G. V., Schwartz, E., Muniz, R. M. \& Meincke, S. M. K. (2011). Modelo bioecológico de Urie Bronfenbrenner e inserção ecológica: uma metodologia para investigar famílias rurais. Texto Contexto-Enfermargem, 20(4), 669-674. 